\title{
镍基单晶高温合金中 $\gamma^{\prime}$ 相筏化行为的相场法研究进展
}

赵彦 $^{(12)}$ ，张洪宇 ${ }^{(1)}$ ，韦华 ${ }^{(1)}$, 郑启 ${ }^{(1)}$, 金涛 ${ }^{(1)}$, 孙晓峰 ${ }^{(1)}$

(1) 中国科学院金属研究所, 沈阳 110016 ;

(2) 上海大学材料科学与工程学院, 上海 200072

* 联系人, E-mail: hwei@imr.ac.cn

2013-05-09 收稿, 2013-08-09 接受, 2013-11-20 网络版发表

国家重点基础研究发展计划(2010CB631200, 2010CB631206)和国家自然科学基金(50931004, 51071164, 51371173, U1037601, 50671102)资助

摘要 形核理论、相图计算(CALPHAD)数据库及微观弹塑性力学的发展, 使镍基单晶高 温合金热处理、疲劳、蠕变等近工况条件下 $\gamma^{\prime}$ 相筏化行为的相场模拟成为可能. 基于镍 基单晶高温合金 $\gamma^{\prime}$ 相筏化行为的实验研究进展，结合相场法对 $\gamma^{\prime}$ 析出及筏化行为的组织 模拟结果, 本文综述了相场模型研究镍基单晶高温合金中 $\gamma^{\prime}$ 箋化行为的主要研究进展.

关键词

单晶高温合金 $\gamma^{\prime}$ 筏化

相场

弹塑性力学
近 30 年来随着数学、物理、计算机科学在材料 领域中的广泛应用, 通过计算模拟研究材料行为已 经能够跨越从电子、原子尺度到连续介质尺度, 并逐 步形成了一些体系较为完善、应用较为广泛的模拟方 法和模型. 理论模型的创新、算法的不断改进以及实 验手段的丰富使得材料的计算模拟越来越贴近真实 的实验过程, 这为从理论上解释一些实验现象 (如 $\gamma^{\prime}$ 筏化和粗化)、优化实验参数、预判材料使役行为和 评价材料力学性能等提供了有力的工具.

镍基高温合金由于具有优异的高温力学性能和 抗氧化腐蚀性能, 广泛用于制备浴轮发动机等先进 动力推进系统的热端部件, 且已成为先进动力推进 系统设计与制造的重大关键技术之一. 镍基单晶高 温合金的微观组织主要由 $\gamma^{\prime}-\mathrm{Ni}_{3} \mathrm{Al}$ 和 $\gamma-\mathrm{Ni}$ 两相组成, 其中 $\gamma$ 是基体相, $\gamma^{\prime}$ 在 $\gamma$ 基体上共格析出, 是最重要的 强化相. 合金服役过程中, 由于温度和应力的协同作 用, 使 $\gamma^{\prime}$ 相沿某个方向发生定向粗化, 形成筏状组织, 显著降低合金高温力学性能, 故 $\gamma^{\prime}$ 筏化是高温合金性 能退化的主要原因之一. 因此, 通过对 $\gamma^{\prime}$ 箋化行为的 理论和实验研究, 对于阐明高温合金的失效机理是 至关重要的. 镍基单晶高温合金 $\gamma^{\prime}$ 相筏化行为的组织 模拟方法主要包括解析 ${ }^{[1]}$ 、相场法 ${ }^{[2]}$ 、Monte Carlo
法 $^{[3]}$ 、有限元法 ${ }^{[4]}$ 等, 其中相场法由于在物理及力学 模型方面的优势, 目前已成为研究 $\gamma^{\prime}$ 相筏化的主要模 拟方法之一. 近些年, 随着相场法对晶体缺陷研究的 逐步深人 ${ }^{[5 \sim 7]}$, 使得 $\gamma^{\prime}$ 相筏化的研究也更加深人.

实验和模拟方法在探讨 $\gamma^{\prime}$ 析出和筏化行为时发 现, $\gamma^{\prime}$ 微观形貌比较复杂: 形貌上, $\gamma^{\prime}$ 有圆形、方形、 枝晶状、片状等 ${ }^{[8,9]}$; 尺寸上, 有过冷条件下析出尺寸 相当的一次 $\gamma^{\prime}$ 相, 也有过冷条件下多次析出尺寸极不 均匀的二次、三次 $\gamma^{\prime}$ 相 ${ }^{[10,11]}$; 此外, 外场作用下相邻 $\gamma^{\prime}$ 相粗化, 或 $\gamma^{\prime}$ 在长大过程中会䢃裂成 3 块、4 块、8 块等 ${ }^{[12,13]}$; 体积分数上, 不同的热处理和外场作用下 $\gamma^{\prime}$ 相可能会产生总体积分数的增加或减少 ${ }^{[14]}$, 这些微 观形态上的复杂性不能够通过某种简单因素作用来 解释, 可能涉及到温度场、塑性流变、力场、有序化、 晶体缺陷等各方面因素的影响.

因此, 发展模拟方法对实验结果进行理论分析, 以及通过组织模拟预测不同服役条件下 $\gamma^{\prime}$ 的析出形 态, 借此来预测其力学性能, 对节约实验成本、优化 工艺、缩短合金研发周期等非常必要. 目前在研究 $\gamma^{\prime}$ 形貌的模拟方法中, 发展最好的是 Monte Carlo 方 法 $^{[2,15]}$ 和相场法 ${ }^{[3,16]}$, 特别是相场法在解释 $\gamma^{\prime}$ 析出行 为及筏化方面已取得了明显进展. 本文通过回顾 $\gamma^{\prime}$ 箋 
化实验和模拟的研究历程, 简要综述近年来相场法 在 $\gamma^{\prime}$ 析出及筏化方面的一些研究结果, 为进一步利用 相场法研究 $\gamma^{\prime}$ 箋化行为提供参考.

\section{1 相场法}

相场法的基本思想是：根据物理模型建立自由 能泛函, 通过变分法求解动力学方程 (如: CahnHilliard 方程、Ginzburg-Landau 方程等), 并以矩阵形 式表示序参数在场中的分布, 从而得到研究对象的 动力学行为. 相场法的优点在于能够通过自由能泛 函的构建, 分别考虑影响研究对象动力学行为的各 种因素, 并动态显示微观组织的演化行为, 从而通过 所建立的模型, 分析导致组织演化行为的内在机制. 相场方法能够研究力场 ${ }^{[14]}$ 、流场 ${ }^{[17]}$ 、材料本征缺陷 ${ }^{[18]}$ 等对组织形貌的影响, 而且在研究枝晶生长取向 ${ }^{[19]}$ 、

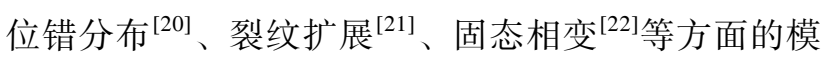
拟结果已得到实验证实.

相场法的应用范围主要包括枝晶生长 ${ }^{[19]}$ 、固态

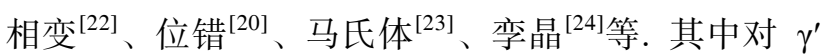
析出及筏化行为的探讨大致经历了从二元到多元, 从弹性模型到弹塑性模型, 从简单的等温过程到复 杂的过冷行为的研究过程. 特别是近几年, 微观弹塑 性力学模型在相场中的应用, 进一步拓展了相场法 的研究范围, 其模拟方法主要包括离散位错方法 ${ }^{[25]}$ 、 有效塑性应变方法 ${ }^{[26]}$ 、黏塑性力学方法 ${ }^{[27,28]}$ 等. 新发 展的相场模型除了关注 $\gamma^{\prime}$ 筏化行为外, 也开始关注缺

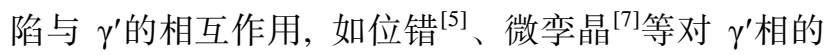
剪切作用, 从而进一步深化对 $\gamma^{\prime}$ 相筏化机理的理解.

\section{2 相场法对 $\gamma^{\prime}$ 析出行为的研究}

相场法研究 $\gamma^{\prime}$ 形貌演化可分为两方面: 一方面 是基于热处理过程中的 $\gamma^{\prime}$ 析出; 另一方面是基于合 金服役过程中 $\gamma^{\prime}$ 的形变. 相场法对热处理过程中 $\gamma^{\prime}$ 析 出行为的研究内容主要包括 $\gamma^{\prime}$ 粗化、多元镍基单晶高 温合金 $\gamma^{\prime}$ 析出和 $\gamma^{\prime}$ 析出的多模态. 其中每个研究内容 又是在相场方法与其他理论模型耦合基础上完成的, 如 $\gamma^{\prime}$ 箋化的相场研究是在微观弹塑性力学及位错理 论基础上发展起来的; 多元镍基基单晶高温合金 $\gamma^{\prime}$ 析出的相场研究是在相场方法和相图计算 (Computer CALculation of PHAse Diagram, CALPHAD)方法耦合基 础上建立起来的; $\gamma^{\prime}$ 析出多模态相场研究是在形核理 论基础上建立起来的. $\gamma^{\prime}$ 筏化的相场研究大致经历了 2 个阶段: 第一个 阶段是 $\gamma^{\prime}$ 筏化的弹性模型; 第二个阶段是 $\gamma^{\prime}$ 筏化的弹 塑性模型. 其中, $\gamma^{\prime}$ 筏化的弹塑性模型又可分为 2 类, 基于位错的弹塑性模型以及晶体塑性模型. 每种模 型均有各自的特点, 弹性模型较容易模拟外加力场、 晶格错配和 $\gamma^{\prime} / \gamma$ 弹性模量差异对 $\gamma^{\prime}$ 筏化行为的影响, 而弹塑性模型中，位错塑性模型则通过模拟位错来 解释 $\gamma^{\prime}$ 筏化行为, 并对 $\gamma$ 通道里的位错类型和分布进 行了图形显示; 晶体塑性模型则是通过模拟 $\gamma^{\prime}$ 筏化 演化过程来解释材料的力学行为. 虽然对 $\gamma^{\prime}$ 筏化的研 究已取得较大进展, 但该研究却一直局限在 $\mathrm{Ni}-\mathrm{Al}$ 二 元合金系.一方面是由于缺少模拟多元合金系所需 的材料参数, 如晶格错配度、不同条件下原子的扩散 系数等; 另一方面是由于现有参数在与相场模型的 匹配性上存在问题，如界面能的计算、计算的收敛性 等方面, 这些都有赖于模型的进一步改进和发展.

在讨论 $\gamma^{\prime}$ 筏化问题之前, 先对多元镍基单晶高 温合金 $\gamma^{\prime}$ 析出及 $\gamma^{\prime}$ 析出的多模态 2 个问题单独进行阐 述, 因为这 2 个问题本身对完善相场法研究 $\gamma^{\prime}$ 筏化具 有重要的推动作用; 之后将从实验和模拟的角度, 重 点从相场法角度阐述 $\gamma^{\prime}$ 筏化的研究进展; 最后对 $\gamma^{\prime}$ 筏 化行为的相场研究提出建议和思考.

\section{1 多元镍基单晶高温合金 $\gamma^{\prime}$ 析出行为的研究进展}

多元镍基单晶高温合金的模拟方法包括第一性 原理 ${ }^{[29]}$ 、Monte Carlo 法 ${ }^{[15]}$ 、相场法 ${ }^{[16,30]}$ 等. 虽然借 助第一性原理和 Monte Carlo 分子动力学有助于获得 合金 $\gamma$ 相和 $\gamma^{\prime}$ 相的晶格常数进而得到两相的晶格错配 度, 并且通过第一性原理还可以计算出合金基态的 弹性常数, 但是, 第一性原理所能计算的体系较小, Monte Carlo 又十分依赖原子间作用势, 它们在多组 元体系计算方面均受到限制. 考虑到高温合金自身 的复杂性, 热动力学模型将大大简化复杂原子构型 沉淀相的模拟. 近十几年来, 计算相图技术的发展以 及镍基高温合金的热动力学数据的积累 ${ }^{[31 ~ 33]}$, 使多 元镍基高温合金 $\gamma^{\prime}$ 析出行为的动力学模拟成为可能.

$\mathrm{Ni}-\mathrm{Al}$ 基单晶高温合金沉淀过程中，在基体 $\gamma$ 相 中析出的 $\gamma^{\prime}$ 相均为面心立方(fcc)结构, $\gamma$ 是以 $\mathrm{Ni}$ 为基 的无序固溶体, $\gamma^{\prime}$ 为 $\mathrm{Ni}$ 占据 $\mathrm{fcc}$ 面心位置、 $\mathrm{Al}$ 占据 $\mathrm{fcc}$ 顶角位置的 $\mathrm{L}_{2}$ 结构有序金属间化合物. 相场模型中 对这两相晶体结构通过 4 个场参数 (1 个成分场参数 和 3 个有序结构场参数 ${ }^{[34]}$ 进行表达. 通过 4 个参数 
所表达的自由能泛函作为相场法中热力学计算的输 人, 求解与成分场演化相关的 Cahn-Hilliard 方程, 以 及有序参数场演化相关的 Ginzburg-Landau 方程所构 成的方程组, 即可得到场参数的演化.

一般情况下自由能泛函的构成主要包括化学自 由能(体自由能)、界面能、弹性能等. 化学自由能密 度曲线为双势阱的形式, 一直以来化学自由能通过 多项式的方式来表达 ${ }^{[22,35]}$, 多项式中的系数通过数 值拟合的方法得到. 多项式对化学自由能表达虽然 成功, 但仅是数学上的表示, 没有涉及材料热力学的 物理本质. 随着相图计算 (Computer CALculation of PHAse Diagram, CALPHAD)方法的发展 ${ }^{[30 \sim 33]}$, 特别 是通过其中的亚晶格模型表示多元合金溶质原子在 晶格位置占位的热力学模型的建立, 借助 CALPHAD 方法得到的热力学数据, 并将其带人到相场模型中, 使得相场法模拟多元合金的相析出行为成为可能. $\mathrm{Zhu}$ 等人 ${ }^{[36]}$ 首次通过把含亚晶格模型的相场方法应 用到 $\mathrm{Ni}-\mathrm{Al}$ 合金 $\gamma^{\prime}$ 的模拟中, 为 CALPHAD 方法与相 场方法耦合模拟 $\gamma^{\prime}$ 析出开辟了先河. Wang 等人 ${ }^{[37]}$ 在 此基础上把集团变分法 (cluster variation method, CVM)计算的界面能、错配度等引人到相场方法中, 模拟了 $\gamma^{\prime}$ 的筏化. 该工作为模拟多元镍基单晶高温合 金 $\gamma^{\prime}$ 的析出提供了铺垫. Kitashima ${ }^{[38]}$ 在 CALPHAD 与 其他计算方法耦合的基础上，提出模拟多元镍基高 温合金 $\gamma^{\prime}$ 析出的相场方法. 虽然 Kitashima 采用的模 型是基于 $Z h u$ 等人 ${ }^{[36]}$ 和 Wang 等人 ${ }^{[37]}$ 的工作，但他将 热力学模型拓展到多元体系, 并在此基础上, 计算了 Ni-Al-Re-Ta 四元系 ${ }^{[39]}$ 和 Ni-Al-Co-Cr-Mo-Re-Ta-W 八元系 ${ }^{[15]}$ 中 $\gamma^{\prime}$ 的析出及合金元素的成分分布和有序 度, 图 1 为 Ni-Al-Co-Cr-Mo-Re-Ta-W 八元合金的相 场模拟结果. 虽然该模型能够获得合金元素的成分 分布和有序度, 但目前只限制在一维条件下的 $\gamma^{\prime}$ 相析
出, 所计算的空间尺度也较小, 并且没有考虑到弹性 场对合金元素扩散的影响.

\section{2 不同过冷条件下的 $\gamma^{\prime}$ 的析出研究}

通常相场法所研究的 $\gamma^{\prime}$ 析出都是在等温条件下 进行的, $\gamma^{\prime}$ 形核过程只有一次, $\gamma^{\prime}$ 相的尺寸相差不大. 在模拟过程中, 通过在方程中加人 Gauss 分布的 Langevin 扰动项使得 $\gamma^{\prime}$ 形核, 扰动项满足涨落-耗散 定律 ${ }^{[40]}$, 并在 $\gamma^{\prime}$ 形核之后去掉 Langevin 扰动项. 该模 拟结果虽然与实际等温情况下的形核过程相近, 但 不能模拟不同过冷条件下的 $\gamma^{\prime}$ 多次析出过程. $\gamma^{\prime}$ 多次 析出 ${ }^{[11,12]}$ 与过冷度和过饱和度密切相关, 与相图中 $\gamma$ 和 $\gamma^{\prime}$ 相线斜率相对应. 为模拟合金时效过程中 $\gamma^{\prime}$ 多次 析出行为, Simmons 等人 ${ }^{[41,42]}$ 通过发展 Poisson 种子 算法, 并将多次形核理论引入到相场模型中. 随后 Wen 等人 ${ }^{[43]}$ 把该算法应用到 $\gamma^{\prime}$ 相模拟中, 计算了 $\gamma$ 通 道中 $\gamma^{\prime}$ 颗粒的二次析出, 研究了不同冷却速度下的 $\gamma^{\prime}$ 析出行为(图 2). Boussinot 等人 ${ }^{[44]}$ 应用该算法, 计算 了多次形核过程中 $\gamma^{\prime}$ 析出的尺寸分布. 通过他们的研 究使得相场法模拟不同冷却速度下的 $\gamma^{\prime}$ 析出行为成 为可能, 为近工程条件下 $\gamma^{\prime}$ 析出行为的模拟提供了重 要参考. 虽然 $\gamma^{\prime}$ 析出的多模态相场研究取得了一些进 展, 但是模型中只讨论了成分场的形核驱动力, 许多 重要的因素(如界面迁移率随温度的变化、不同冷速 对 $\gamma^{\prime}$ 颗粒生长各向异性的影响等)没有考虑进去, 不 过这些研究进展依然为近工程条件下 $\gamma^{\prime}$ 析出行为的 模拟研究迈出了重要的一步.

\section{$3 \gamma^{\prime}$ 筏化的研究进展}

\section{1 实验研究进展}

$\gamma^{\prime}$ 是镍基单晶高温合金中最重要的强化相, 其筏 化行为能显著影响合金的综合高温力学性能. 因此,

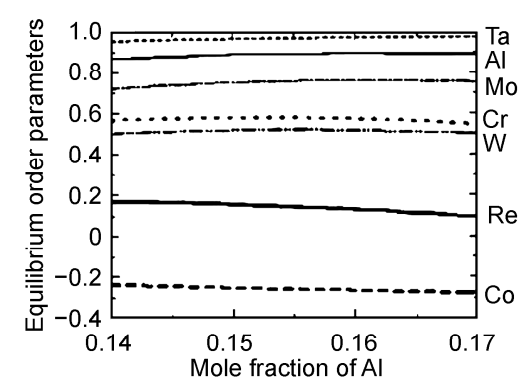

(a)

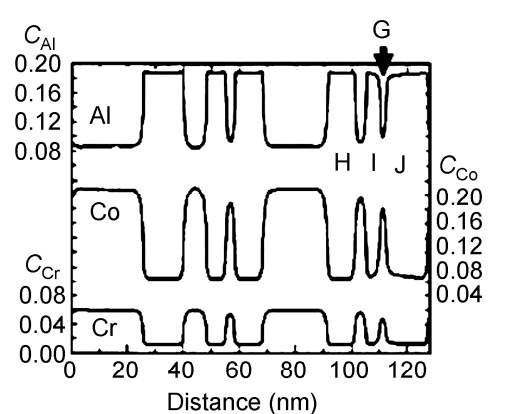

(b)

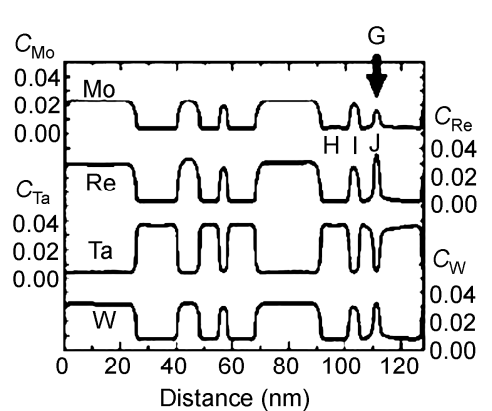

(c)

图 1 TMS-75 合金 $1100{ }^{\circ} \mathrm{C}$ 时效过程中, 合金元素在 $\gamma^{\prime}$ 相中的(a)序参数平衡值(即有序度)以及 $((\mathbf{b}),(\mathrm{c}))$ 成分分布 ${ }^{[15]}$ 

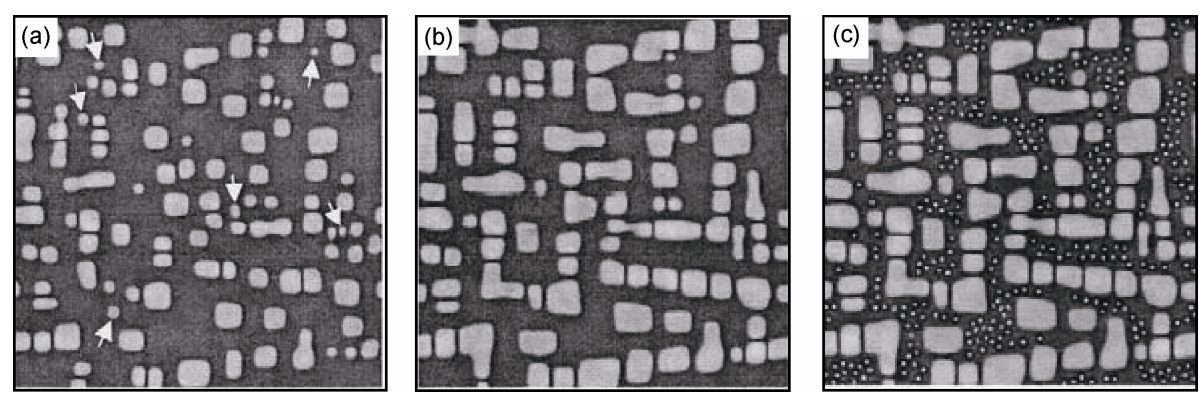

图 $21 K / \tau$ 冷速下的 $\gamma^{\prime}$ 析出形貌演化图 ${ }^{[43]}$

(a) $\tau=40$; (b) $\tau=150$; (c) $\tau=450 . \tau$ 为约化时间步数

$\gamma^{\prime}$ 箋化的研究备受关注. 无论实验还是模拟研究, $\gamma^{\prime}$ 筏化的研究主要集中于 $\gamma^{\prime}$ 形筏取向和筏化的驱动力, 导致它们发生变化的因素主要包括: 外加应力、晶格 错配及 $\gamma^{\prime}$ 相与 $\gamma$ 相弹性模量差. 此外, 应变能的释放, 特别是位错对 $\gamma^{\prime}$ 箋化的影响也至关重要.

$\gamma^{\prime}$ 筏化的实验研究开始较早, 1971 年 Tien 和 Copley ${ }^{[45]}$ 首次研究了 [001]取向镍基单晶合金中的 $\gamma^{\prime}$ 形筏现象, 发现 $\gamma^{\prime}$ 形筏不仅改变 $\gamma^{\prime}$ 形貌, 更重要的是, 能显著影响 $\gamma / \gamma^{\prime}$ 界面位错网形成及合金元素在该界面 的分布, 故对合金力学性能具有重要影响 ${ }^{[46]}$. Pineau ${ }^{[47]}$ 利用 Eshelby 理论预测了单轴应力条件下的 $\gamma^{\prime}$ 筏化, 并提出了著名的筏化预言图. 该图表示了在 纯弹性方法和各向同性近似条件下，外加应力、晶格 错配及 $\gamma$ 基体和 $\gamma^{\prime}$ 析出相弹性模量差异对筏化方向的 影响, 并强调 $\gamma^{\prime}$ 筏化方向取决于 $\gamma^{\prime}$ 析出相与 $\gamma$ 基体间 弹性常数差 $\left(c_{11}{ }^{-} c_{12}\right)$ 之间的比值. Carry 和 Strude ${ }^{[48]}$ 在研究单晶合金蠕变性能基础上, 推测 $\gamma / \gamma^{\prime}$ 界面处的 位错可能通过攀移沿着 $\gamma^{\prime}$ 颗粒的边界运动, $\gamma^{\prime}$ 筏化方 向与 $\gamma / \gamma^{\prime}$ 界面位错分布密切相关, 即在 $\gamma^{\prime}$ 定向形筏过 程中未消失的 $\gamma / \gamma^{\prime}$ 界面是被塑性流变位错覆盖的界面, 表明塑性变形是导致 $\gamma^{\prime}$ 定向形筏的关键因素. Miyazaki 等人 ${ }^{[49]}$ 将 $\gamma^{\prime}$ 立方块的各向异性考虑到分析中, 通 过能量计算与颗粒尺寸的比较研究了 $\gamma^{\prime}$ 筏化方向. Fredholm 和 Strudel ${ }^{[50]}$ 根据筏状 $\gamma^{\prime}$ 的不同特征将其分 为垂直于外加应力方向的 $\mathrm{N}$ 型筏和平行于外加应力 方向的 $\mathrm{P}$ 型筏, 其中 $\mathrm{N}$ 型筏可能降低合金疲劳性能, $\mathrm{P}$ 型筏可能改善合金疲劳性能 ${ }^{[51]}$. Nathal 等人 ${ }^{[52]}$ 和 Schneider 等人 ${ }^{[53]}$ 在研究 CMSX-4 合金高温蠕变性能 时指出, $\gamma^{\prime}$ 形筏后改变了 $\gamma$ 与 $\gamma^{\prime}$ 连接方式, 使 $\gamma$ 基体由包 围着 $\gamma^{\prime}$ 变为镶嵌在 $\gamma^{\prime}$ 中, 从而失去变形能力而易于断 裂, 故 $\gamma^{\prime}$ 形筏会不同程度地降低合金蠕变强度. $\gamma^{\prime}$ 形
筏源于应力梯度导致合金元素的定向扩散, 即在应 力梯度作用下, $\gamma^{\prime}$ 相形成元素 ( $\mathrm{Al}, \mathrm{Ti}$ 和 $\mathrm{Ta}$ 等)和 $\gamma$ 相形 成的元素 $\left(\mathrm{Cr}, \mathrm{Mo}\right.$ 等)沿相反方向扩散, 致使 $\gamma^{\prime}$ 沿特定 方向生长且互相连接, 最终导致 $\gamma^{\prime}$ 形筏, 且因 $\gamma^{\prime}$ 形筏 过程主要受固相扩散控制, 故其 $\gamma^{\prime}$ 形筏动力学呈非线 性特征 ${ }^{[8,54]}$. Pollock 和 Argon ${ }^{[55]}$ 在研究塑性变形和位 错对 $\gamma^{\prime}$ 形筏作用时指出, $\gamma^{\prime}$ 定向形筏是通过 $\gamma^{\prime}$ 颗粒局部 溶解和合金元素定向扩散实现的, 合金元素对蠕变 过程中 $\gamma^{\prime}$ 形貌演化的影响主要表现在对 $\gamma / \gamma^{\prime}$ 晶格错配 度和元素扩散速率的影响上, 其中 $\gamma / \gamma^{\prime}$ 界面共格应力 是 $\gamma^{\prime}$ 定向形筏的主要驱动力, 同时蠕变过程中的位错 攀移和滑移也促进合金蠕变, 进而加速 $\gamma^{\prime}$ 形筏 ${ }^{[56]}$. 尽 管位错滑移后期形成的筏排组织封闭了位错运动的 横向通道, 增加了蠕变抗力, 但形筏毕竟是 $\gamma^{\prime}$ 粗化的 结果, 所以大多情况下对合金性能具有不利影响。 Ignat 等人 ${ }^{[57]}$ 测试了 $\gamma^{\prime}$ 筏化与应力状态的关系并指出, 未释放的晶格错配所驱动的合金原子扩散削弱了错 配位错的相互作用，对于 $\mathrm{N}$ 型筏化，合金原子扩散在 平行于应力方向的 4 个面被激活; 对于 $\mathrm{P}$ 型筏化, 在 垂直于应力方向的 2 个相反的面被激活. Matan 等 人 $^{[58]}$ 提出当塑性临界应变值超过 $0.1 \%$ 时, 即使没有 施加应力的试样, $\gamma^{\prime}$ 筏化仍将继续进行, 即当塑性应 变达到临界应变值时, $\gamma$ 通道内有较多的位错, 但位 错之间相互缠结使得位错可动性变差，当去掉外加 应力时，位错聚集在 $\gamma / \gamma^{\prime}$ 界面而不能消失，故通过位 错释放晶格错配使 $\gamma^{\prime}$ 筏化能够继续进行. Fahrmann 等 人 ${ }^{[59]}$ 通过实验测试 $\gamma^{\prime}$ 筏化方向与 $\gamma$ 基体和 $\gamma^{\prime}$ 析出相弹 性模量差之间的关系指出，对于弹性模型， $\gamma$ 与 $\gamma^{\prime}$ 弹性 模量差的正负将决定 $\gamma^{\prime}$ 的筏化方向, 且 $\gamma^{\prime}$ 筏化方向依 赖于 $\sigma\left(E_{\gamma^{\prime}}-E_{\gamma}\right) / \delta\left(E_{\gamma}\right)^{2}$, 其中 $\sigma$ 为应力大小, $\delta$ 为各向异 性常数, $E_{\gamma^{\prime}}$ 与 $E_{\gamma}$ 分别为 $\gamma^{\prime}$ 相和 $\gamma$ 相的弹性模量; 而对弹 
-塑性模型, $\gamma^{\prime}$ 筏化方向只依赖于 $\sigma / \delta E_{\gamma}$.

Paris 等人 ${ }^{[8]}$ 观察到 $\gamma^{\prime}$ 筏化与位错网的形成是同 时开始的, 表明 $\gamma^{\prime}$ 筏化的塑性驱动机制, 且这种滑移 位错择优处于水平 $\gamma$ 通道内, $\gamma^{\prime}$ 筏化行为由 3 个非线性 行为组成. 第一阶段为均匀增加阶段; 第二阶段为快 速增加阶段. 在扩散流作用下, 单个析出相 $\gamma^{\prime}$ 形成元 素 $\mathrm{Al}, \mathrm{Ti}$, Ta 再分配所导致, 此时 $\gamma^{\prime}$ 析出相的扩散尺 度大约是 $100 \mathrm{~nm}$; 第三阶段中, 由于驱动力减少, $\gamma^{\prime}$ 筏化速度减慢, $\gamma / \gamma^{\prime}$ 界面位错的出现改变了溶质原子 的化学势梯度, 与连续的 $\gamma / \gamma^{\prime}$ 界面状态相比, $\gamma / \gamma^{\prime}$ 界面 位错对 $\gamma^{\prime}$ 筏化过程至关重要. 从实验上讲, 完整连续 的 $\gamma / \gamma^{\prime}$ 界面很难在长时间退火过程中或应力低于 40 $\mathrm{MPa}$ 的情况下保持, 这可能是由于 $\gamma$ 通道内元素的非 平衡分布和加热状态有利于位错运动所致.

\section{2 计算机模拟研究进展}

通过计算机模拟研究 $\gamma^{\prime}$ 筏化行为主要考虑两方 面问题，一方面是基于方法的选择，如有限元 ${ }^{[60] 、}$ Monte Carlo 方法 ${ }^{[61]}$ 、相场方法 ${ }^{[37]}$ 等; 另一方面是基

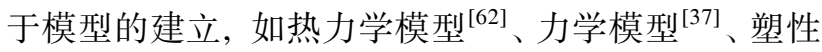
流变模型 ${ }^{[25]}$ 等.

Socrate 和 Parks ${ }^{[4]}$ 基于弹性能量分析, 在有限元 模拟基础上指出, $\gamma^{\prime}$ 形筏驱动力正比于外加应力、 $\gamma / \gamma^{\prime}$ 错配度大小以及 $\gamma / \gamma^{\prime}$ 两相弹性常数之差, 且 $\gamma^{\prime}$ 形筏能 明显降低系统的能量. Matan 等人 ${ }^{[58]}$ 考虑到塑性变形 对 $\gamma^{\prime}$ 形筏过程的影响, 借助于塑性变形模型, 通过数 值模拟得到, $\gamma^{\prime}$ 形筏必然伴随塑性应变, 但 $\gamma^{\prime}$ 要显著 形筏必须超过其临界应变量. 在此基础上, Buffiere 和 Ignat ${ }^{[63]}$ 提出了 $\gamma^{\prime}$ 形筏的位错判断准则, 即位错首 先出现在能使 $\gamma / \gamma^{\prime}$ 错配应力松弛的 $\gamma$ 通道中, 且在蠕变 初始阶段, 通过 $\gamma / \gamma^{\prime}$ 界面扩展来降低其界面错配能, 促进 $\gamma^{\prime}$ 形筏, 进而降低整个系统的能量, 因此, 系统 能量降低是 $\gamma^{\prime}$ 形筏的根本动力. 此外, Osawa 等人 ${ }^{[60]}$ 通过有限元模拟发现，材料中位错分布有利于优化 $\gamma$ 通道的方向.

Véron 等人 ${ }^{[46]}$ 利用三维模拟描述了 $\gamma^{\prime}$ 筏化过程的 形貌和动力学行为, 认为蠕变过程中由位错所产生 的各向异性晶格错配的松驰是筏化的驱动力, 并且 计算了由成分梯度和塑性流变所导致的扩散流，其 中局部应力通过宏观外加应力和错配应力(它们依赖 于位错的分布)来获得. 计算发现, 当由塑性应变导 致的晶格错配被释放时, 大部分受载的 $\gamma$ 通道将首先
经历塑性, 位错择优处于垂直于应力轴的通道界面. 实验结果显示, 拉伸实验中负错配度情况和压缩实 验中正错配度情况下, 其实验结果和模拟结果相符, 即被位错填充的择优通道不仅依赖于载荷方向, 也 依赖于晶格错配符号. Véron 等人 ${ }^{[46]}$ 同时认为, 弹性 能密度的非均匀性驱动了不同类型通道的扩散流, 模拟结果显示, 对于拉伸实验, $\gamma^{\prime}$ 以 $\mathrm{N}$ 型筏化; 对于 压缩实验, $\gamma^{\prime}$ 以 $\mathrm{P}$ 型筏化. 在更高的应力条件下, $\gamma^{\prime}$ 筏 化将加速, 压缩过程中 $\gamma^{\prime}$ 箋化速度比拉伸过程慢 4 倍. 在此基础上, Véron 等人 ${ }^{[46]}$ 通过图表展示了 $\gamma^{\prime}$ 筏化方 向与晶格错配和外加应力之间的关系，遗憾的是，该 图在正错配度区域不能由实验证明，其原因可能是 Véron 模型过多强调了塑性流对 $\gamma^{\prime}$ 筏化方向的影响. 进一步分析也表明，在正错配度区域塑性流与原子 扩散流作用相反, 实际上许多蠕变实验也证实, 只有 当蠕变应变达到 $0.2 \%$ 时, $\gamma^{\prime}$ 筏化才完成. 因此, 与原 子扩散流对 $\gamma^{\prime}$ 筏化的影响相比, 塑性流的影响不是最 重要的.

Nabarro ${ }^{[62]}$ 提出一个计算 $\gamma^{\prime}$ 筏化热力学压力的物 理模型, 获得的结果表明, 当塑性流变发生时, $\gamma^{\prime}$ 和 $\gamma$ 相弹性常数之间的差异对 $\gamma^{\prime}$ 筏化的影响很小; 外加应 力场只增加 $\gamma$ 相的自由能, 通过计算焓变与 $\gamma / \gamma^{\prime}$ 界面 的位移, 得出初始的化学热力学压力相当于 20 倍的 纯弹性效应的热力学压力. 同时, 由体自由能引起的 应力作用下的界面迁移可以忽视，由体自由能引起 的早期大的热力学压力不能对抗弹性效应的热力学 压力 (其随着界面迁移保持为常数), 因此, 实验很难 证实该模型的结果. Nabarro 的模型也只考虑了一个 $\gamma$ 通道和 $\gamma^{\prime}$ 相之间的平衡，事实上不同种类的 $\gamma$ 通道之 间自由能的差异将使原子扩散流连续并且使 $\gamma / \gamma^{\prime}$ 界面 向前移动, 另外, 仅从塑性的角度而言, 在一定应力 条件下 $\gamma$ 相体自由能变化的方向对垂直和平行于应 力方向的通道是不同的. 如果假定局部晶格错配和 溶质成分呈线性关系的话, Nabarro 模型中的一些推 导将值得商榷.

Arrell 和 Vallés ${ }^{[64]}$ 在计算 $\gamma^{\prime}$ 相每个立方面自由能 密度基础上，通过对比能量密度来预测 $\gamma^{\prime}$ 筏化的形貌, 但该模型没有考虑蠕变应力和 $\gamma^{\prime}$ 筏化过程中载荷的 作用，获得的界面能来自于界面位错对界面能的贡 献. 模拟结果与负错配度合金中 $\gamma^{\prime}$ 筏化结果一致, 并 解释了在 $E_{\gamma^{\prime}}>E_{\gamma}$ 情况下 $\gamma^{\prime}$ 筏化的原因. Vallés 和 Arrell ${ }^{[65]}$ 也通过 Monte Carlo 模拟再现了不同应力和 
温度条件下的 $\gamma^{\prime}$ 筏化，但是该模型仍旧建立在不同密 度的界面位错所引起的界面能的基础上, 界面完全 释放由晶格错配和载荷引起的应力.

Ohashi 等人 ${ }^{[66,67]}$ 根据 $\gamma$ 通道内塑性应变分布，借 助几何位错推导出了位错密度公式. 随后通过考虑 外加应力、晶格错配和位错所得到的应变能, 获得了 由 $\gamma^{\prime} / \gamma$ 界面纯刃型位错所建立的水平和垂直 $\gamma$ 通道的 应力场. 水平和垂直 $\gamma$ 通道的应变能密度差大约是 2 $\mathrm{mJ} / \mathrm{m}^{3}$, 足以引起 $\gamma^{\prime}$ 筏化的扩散. 模型尝试把塑性变 形和几何位错分布连接起来, 仅通过观察 Von-Mises 应力分布来分析 $\gamma^{\prime}$ 筏化更合理，但该模型仅考虑 了 [001]晶向排列的刃型位错, 有一定的局限性.

Nabarro 等人 ${ }^{[1]}$ 考虑到单晶材料各向异性, 借助 Eshelby 能量-动量张量分析方法得到了 $\gamma^{\prime}$ 筏化驱动力 的解析解. 结果表明, $\gamma^{\prime}$ 筏化驱动力正比于拉力、晶格 错配和 $\gamma^{\prime} / \gamma$ 弹性模量差; 当塑性应变超过一定值后, 应力方向决定 $\gamma^{\prime}$ 筏化方向, $\gamma^{\prime}$ 析出相和 $\gamma$ 基体的弹性模 量之差是次要因素, 这与 Socrate 和 Parks ${ }^{[4]}$ 的 $2 \mathrm{D}$ 分 析结果一致. 同时 Nabarro 等人 ${ }^{[1]}$ 也强调了蠕变过程 中位错的重要性, 证明如果只考虑弹性作用, 在基体 和析出相弹性常数相同情况下, 外加应力不会驱动 $\gamma^{\prime}$ 筏化, 即 $\gamma^{\prime}$ 筏化实际上是由外加载荷和内能增加之间 的差异来驱动的.

\section{3 相场弹性模型研究}

相场法对 $\gamma^{\prime}$ 箋化行为的研究, 应该追溯到微观弹 性理论的建立, 在 Khachaturyan ${ }^{[68]}$ 的书中有详细总 结. 通过微观弹性理论能够解释弹性场与 $\gamma^{\prime}$ 析出相形 貌之间的关系 ${ }^{[69]}$, 在此基础上，考虑到析出相和基 体相之间弹性常数差异引起的非均匀弹性对析出相

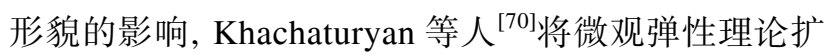
展到非均匀弹性情况, 非均匀弹性公式的推导为相 场法模拟弹性情况下 $\gamma^{\prime}$ 筏化行为提供了理论依据. 在 非均匀弹性公式推导基础上, Chen 课题组 ${ }^{[71,72]}$ 发展 了计算非均匀弹性的扰动迭代方法 (perturb-iterative method)和共轭梯度方法(conjugate gradient method), 显著推动了高阶精度的弹性场数值算法的研究进展; 同时提出了求解相场动力学方程的半隐 Fourier 谱算

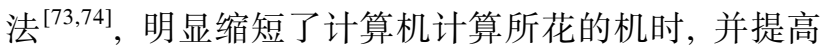
了计算精度. Wang 等人 ${ }^{[34,75]}$, Rubin 和 Khachaturyan ${ }^{[22]}$ 通过在相场模型中引人序参数，且考虑到合金原子 的有序化, 使 $\gamma^{\prime}$ 析出过程中形貌的演化更接近实验所
观察的结果, 并成功解释了 $\gamma^{\prime}$ 析出过程中所发生的䢃 裂现象 ${ }^{[13]}$, 深人探明了有序化对 $\gamma^{\prime}$ 析出相形貌的影响, 指出 $\gamma^{\prime}$ 在反相畴界能大于界面能 0.5 倍时将发生䢃裂. $\mathrm{Li}$ 和 $\mathrm{Chen}^{[76]}$ 基于非均匀弹性理论, 研究了外部载荷 作用下 $\gamma^{\prime}$ 相的筏化行为. 结果显示, 外加力场方向对 $\gamma^{\prime}$ 箋化方向的影响至关重要, 这与拉伸和压缩条件下 的 $\gamma^{\prime}$ 箋化结果一致. 此外, Yang 等人 ${ }^{[77]}$ 和 Wang 等 人 $^{[37]}$ 也分别通过相场方法对外加载荷作用下的 $\gamma^{\prime}$ 筏 化方向进行了研究.

$\gamma^{\prime}$ 筏化的弹性模型在研究应力条件下 $\mathrm{P}$ 型和 $\mathrm{N}$ 型 $\gamma^{\prime}$ 筏化形貌特征时，不仅模拟结果得到了实验证实, 且考虑到外加应力、晶格错配、 $\gamma / \gamma^{\prime}$ 晶格弹性模量差 异对 $\gamma^{\prime}$ 筏化的影响, 故弹性模型在研究 $\gamma^{\prime}$ 筏化方面具 有明显的优势. 值得注意的是, 相场法的弹性模型把 $\gamma^{\prime}$ 筏化看成是应力诱导扩散的过程, 所以没有考虑晶 体缺陷, 尤其是位错对 $\gamma^{\prime}$ 箋化的影响. 正如 Véron 和 Bastie $\mathrm{e}^{[78]}$ 实验结果显示, $\gamma^{\prime}$ 筏化过程不仅要考虑原子 扩散流, 还要考虑塑性流变的影响, 因此有必要发展 $\gamma^{\prime}$ 筏化的弹塑性模型.

\section{4 相场法研究 $\gamma^{\prime}$ 筏化的弹塑性模型}

实验中发现，在没有外加应力的作用下，当施加 较大的变形时, $\gamma^{\prime}$ 也会发生自发筏化 ${ }^{[46,78]}$, 这表明 $\gamma^{\prime}$ 筏化本身与材料的塑性紧密相关. 近几年随着相场 理论的发展, 开始引入相场的弹塑性模型 ${ }^{[10,25,79]}, \gamma^{\prime}$ 筏化的弹塑性模型也由此得到发展. 现阶段通过相 场法研究 $\gamma^{\prime}$ 筏化的弹塑性模型分为 3 种: 离散位错方

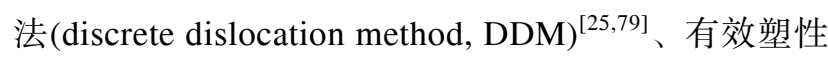
应变方法 ${ }^{[10]}$ 和黏塑性方法 ${ }^{[11,14]}$. 其中, 前 2 种模型属 于 $\gamma^{\prime}$ 筏化的位错模型, 从微观机制方面解释 $\gamma^{\prime}$ 筏化行 为; 后 1 种模型属于 $\gamma^{\prime}$ 箋化的黏塑性力学方法, 该方 法将从宏观力学实验获得的参数引人到相场模型中, 借此来研究 $\gamma^{\prime}$ 筏化行为.

实验证实, 位错与 $\gamma^{\prime}$ 筏化密切相关, 且对 $\gamma^{\prime}$ 箋化 有重要影响, Yeh 等人 ${ }^{[80]}$ 发现沿[001]方向施加应力, 一段时间后位错将聚集在 $\langle 110\rangle$ 方向的 $\{001\} \gamma / \gamma^{\prime}$ 界面. Pollock 和 Argon ${ }^{[55]}$ 发现对于负错配度的单晶高温合 金, 低温高应力拉伸时, 位错更倾向于沿着水平 $\gamma$ 通 道进行滑移; 高温低应力拉伸时, 在平行于拉伸轴的 $\gamma$ 通道内几乎没有位错的存在. Tetzlaff 和 Mughrabi ${ }^{[81]}$ 工作进一步表明, 当水平 $\gamma$ 通道的位错到达 $\gamma^{\prime}$ 颗粒边 界时, 它们可能划过垂直通道, 如果局部应力足够大, 
在 $\gamma / \gamma^{\prime}$ 垂直通道内将建立一定的位错排列.

Lahrman 等人 ${ }^{[82]}$ 通过透射电子显微镜(TEM) 观 察到 $\gamma^{\prime}$ 周围位错网中 $1 / 2\langle 110\rangle$ 刃型位错, Singh 等人 ${ }^{[83]}$ 发现 $\gamma^{\prime}$ 周围有绕过-滑移和攀移的位错网络重排, 也 肯定了 $\gamma$ 周围 $1 / 2\langle 110\rangle$ 刃型位错占优的特征. Reed 等 人 ${ }^{[84]}$ 通过 TEM 观察也提出所谓的多边形和方形位错 网, 并在实验中发现, CMSX-4 合金在 $1150^{\circ} \mathrm{C} / 100$ $\mathrm{MPa}$ 条件下蠕变 $10 \mathrm{~h}$ 后 $\gamma^{\prime}$ 快速筏化, 并观察到蠕变硬 化现象. 蠕变速率降低的可能原因是由于 $\gamma^{\prime}$ 筏状结构 的形成, 减小了垂直通道宽度, 阻碍了 $\{111\}\langle 110\rangle$ 位 错的滑移和攀移. Reed 等人 ${ }^{[84]}$ 的实验结果与 Lahrman 等人 ${ }^{[82]}$ 对位错网络的解释不同, Lahrman 等人 ${ }^{[82]}$ 认为 位错的形成是由于 [001]蠕变载荷, 而不是过时效热处 理, 虽然两者均出现了相似的位错网络, 但 Lahrman 等人 ${ }^{[82]}$ 证明, 过时效试样的位错网可能与外加应力 下形成的位错网是不同的. Pollock 和 Argon ${ }^{[56]}$ 在研究 $850^{\circ} \mathrm{C} / 552 \mathrm{MPa} \mathrm{CMXS}-3$ 合金蠕变行为时, 将其蠕变 过程分为 3 个阶段: 孕育期阶段, 即有初生位错增加; 随后位错网络开始形成; 最后位错切过 $\gamma^{\prime}$ 相导致合金 失效, 并解释了各个阶段位错的形貌和演化过程. Keller 等人 ${ }^{[85}$ 通过 TEM 分析 $\langle 001\rangle$ 拉伸蠕变 $\gamma / \gamma^{\prime}$ 界面 位错网络发现, 位错位于平行于蠕变应力的析出面, 垂直于蠕变应力面上的位错释放黏性应变, 从而加 剧黏性应变. Probst-Hein 课题组 ${ }^{[86,87]}$ 借助离散位错方 法(DDM)估算不同 $\gamma$ 通道中的位错密度, 借此来研究 位错密度对 $\gamma^{\prime}$ 体积分数和晶格错配的影响. 结果表明, 晶格错配将吸收位错环, 同时使相同的位错环难以 进入另一种 $\gamma$ 通道. 在此基础上, 通过相场方法模 拟 ${ }^{[8]}$, 解释了由于晶格错配和外部载荷方向对位错 通道的择优性选择.

相场位错方法首先由 Léonard 和 Desai ${ }^{[89]}$ 提出, 但对 $\gamma^{\prime}$ 筏化的研究则起源于 DDM 方法的建立 ${ }^{[25,79,90]}$. 相场位错方法首先用到解释 $\gamma^{\prime}$ 筏化, 最初的想法是通 过把位错方法和溶质扩散 2 种相场方法进行耦合, 从 而形成了相场的离散位错方法. Zhou 等人 ${ }^{[25]}$ 最早提 出计算 $\gamma^{\prime}$ 筏化的离散位错方法, 通过溶质再分配的化 学梯度势来得到位错构型和溶质再分配, 其中溶质 再分配的化学梯度势由 $\gamma / \gamma^{\prime}$ 微观结构、通道位错、外 加应力 3 部分组成, $\gamma / \gamma^{\prime}$ 微观结构和外加应力控制位 错的演化. 该方法把溶质场和位错场同时考虑到相 场模型中, 溶质场中体自由能和界面能与一般的相 场模型相同, 位错场通过多个序参数表达, 并考虑每
个滑移系对系统能量的贡献. 此外, 总的弹性应变能 由内应力、通道位错所形成的应力场和外加应力 3 部分组成. 借助该模型可以得到 $\gamma$ 通道中位错的构 型、密度以及溶质原子的再分配. 该模型的优点是把 位错对筏化的贡献考虑进模型, 并能通过模拟计算 出 $\gamma$ 通道中位错的构型和密度, 但目前该模型没有考 虑有序化和多种滑移系对 $\gamma^{\prime}$ 筏化的影响, 所计算的位 错构型比较简单. 图 3 为通过该方法模拟得到结果, 图 3(b)显示了 $\gamma^{\prime}$ 最终连接成板条状, 即 $\mathrm{N}$ 型筏化. 该 模型主要关注的是每个位错对 $\gamma^{\prime}$ 筏化的贡献, 故称此 方法为离散位错方法. 利用该方法的模拟结果, 证实 了 $\gamma^{\prime}$ 筏化与 $\gamma^{\prime} / \gamma$ 界面位错的类型和分布有关, 同时晶 格错配度符号和载荷方向决定 $\gamma$ 通道中位错的构型.

Zhou 等人 ${ }^{[26]}$ 建立了另一种模拟 $\gamma^{\prime}$ 筏化的弹塑性 模型, 即有效塑性应变方法. 但该模型没有考虑每个 位错对 $\gamma$ 通道塑性应变的贡献, 而是将从实验获得的 位错密度带人到相场模型中, 用等效应变作为 $\gamma$ 通道 的塑性应变. 有效塑性应变方法与离散位错方法的 差异在于, 有效塑性应变方法不考虑单个位错的作 用, 因此不能表示位错的构型和排列; 有效塑性应变 方法仅把位错对 $\gamma$ 通道塑性应变考虑到自由能泛函中, 没有考虑它对体自由能及界面能的影响. 但该模型 的优点在于模型简单, 容易分析 $\gamma$ 通道塑性应变对筏 化的影响. Zhou 等人 ${ }^{[26]}$ 利用有效塑性应变方法分析 了 $\gamma^{\prime}$ 和 $\gamma$ 相弹性模量差异和通道塑性位错对 $\gamma^{\prime}$ 筏化的 影响, 结果表明, 在 $\gamma^{\prime}$ 筏化初期 $\gamma^{\prime}$ 和 $\gamma$ 相弹性模量的差 异驱动 $\gamma^{\prime}$ 筏化, 到后期主要是通道中位错所导致的塑 性驱动 $\gamma^{\prime}$ 筏化, 同时模拟显示 $\mathrm{P}$ 型筏化比 $\mathrm{N}$ 型筏化速 度快.

相场黏塑性模型是一种模拟 $\gamma^{\prime}$ 筏化的唯象模型, 最早由 Ubachs 等人 ${ }^{[91]}$ 在研究焊料合金黏塑性行为时 提出. Uehara 等人 ${ }^{[92]}$ 和 Guo 等人 ${ }^{[93]}$ 分别通过弹塑性
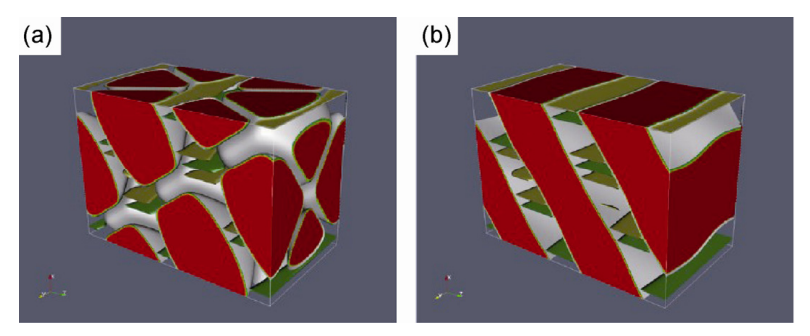

图 $30.3 \%$ 晶格错配 $152 \mathrm{MPa}[001]$ 方向拉伸下 $\gamma^{\prime}$ 相 $\mathrm{N}$ 型筏化 的演化过程 ${ }^{[25]}$

(a) $t=7.2 \mathrm{~h}$; (b) $t=10.7$ h. $\gamma$ 通道中的阴影面为位错的滑移面 
模型研究了晶粒的生长行为和缺陷周围的应力分布; Gaubert 等人 ${ }^{[14]}$ 利用黏塑性模型探讨了高温合金蠕变 过程中的 $\gamma^{\prime}$ 筏化行为. 该模型获得的 $\gamma^{\prime}$ 筏化形貌与实 验结果一致, 解释了反向畴界粗化, 阐明了合金蠕变 过程中应变取向的规律. 该模型的基本思想是: 在相 场弹性模型基础上, 不改变体自由能和界面能, 但微 观力场对自由能的贡献被划分为弹性能和黏塑性能 2 部分, 将其引人相场模型来探究 $\gamma^{\prime}$ 箋化过程中成分 场、有序参数场以及应力场的影响. 模型中的弹性应 变由总应变量减去内应力引起的应变和塑性应变引 起的应变量之和得到, 根据弹性应变得到弹性应变 能, 其中塑性应变以切应变引起的晶体滑移组成, 滑 移系在镍基单晶高温合金中由 12 个八面体、12 个十 二面体、6 个六面体滑移系组成, 在目前的研究中只 考虑了八面体的 12 个滑移系, 切应变由 Norton 流法 则得到, 并考虑到等向强化和随动硬化对剪切应变 的影响. 黏塑性能只考虑随动硬化对自由能的影响. 由于该模型是唯象模型, 模型中选取的参数依赖于 蠕变和疲劳实验或者有限元计算. 该模型的优点在 于与实验结果符合很好, 且能够把宏观加载与 $\gamma^{\prime}$ 筏化 行为相结合, 但其模拟结果在很大程度上依赖于唯 象参数的选取, 并且由于该模型基于相场连续模型, 所以蠕变条件下塑性应变只能模拟蠕变的前 2 个阶 段, 此外该模型在解释筏化微观机制方面不如位错 模型好. 图 4 给出了不同相场模型对 $\gamma^{\prime}$ 形貌及与蠕变 实验结果的对比, 不难看出, 相场弹塑性模型得到的 $\gamma^{\prime}$ 筏化形貌的模拟结果与实验结果匹配更好, 且形貌 非常相近, 故证明了该模型的正确性.

\section{4 相场法研究 $\gamma^{\prime}$ 筏化的展望}

尽管相场方法在研究镍基单晶高温合金中 $\gamma^{\prime}$ 筏
化方面取得明显进展, 但通过相场法研究 $\gamma^{\prime}$ 箋化目前 还主要处于热力学和力学模型的建立阶段. 作者认 为, 多元合金热动力学数据的积累是相场法工程应 用的基础; 同时, 非平衡热力学条件在相场方程和自 由能泛函中表达形式的优化对模拟复杂热处理条件 下的 $\gamma^{\prime}$ 析出行为也十分重要; 此外, 针对不同成分合 金, 获得如 $\gamma$ 和 $\gamma^{\prime}$ 相晶格常数及弹性常数、位错运动 及其相互作用机理等微纳观尺度的结构和力学信息, 对相场法研究 $\gamma^{\prime}$ 箋化行为至关重要. 因此, 通过相场 法实现工程应用中 $\gamma^{\prime}$ 筏化行为的再现, 仍旧是较为长 远的过程, 需要数据的积累和模型的优化. 为此需要 面对以下的挑战:

(1) 完善平衡热力学数据库, 进一步改进非平衡 热力学模型. 目前通过相场方法研究非平衡条件下 的相场热动力学模型, 仅处于模型简单修正阶段, 还 没有从根本上建立适合于相场模拟的非平衡热力学 模型.

(2) 多元合金弹塑性模型的建立. 不同合金元素 对 $\gamma^{\prime}$ 筏化过程中晶格错配度、界面能释放、位错阻碍、 基体塑性流变等的影响是不同的, 有必要把这些因 素综合考虑到弹塑性模型中, 从而改进相场模型.

（3）扩散流场下 $\gamma^{\prime}$ 生长行为的模拟. $\gamma^{\prime}$ 的形貌不 单是球形、立方形, 还会有枝晶状, 说明 $\gamma^{\prime}$ 的生长与 扩散流密切相关, 现阶段流场模型应用到凝固过程 的枝晶生长中, 是否也可以应用到固态相变中, 有待 进一步商榷.

通过前人研究工作的总结, 提以下几点工作展望:

(1) 多元镍基单晶高温合金二维、三维的相场 模拟. 现阶段多元镍基单晶高温合金的相场模拟 仅能模拟一维情况, 高维模拟还没有实现, 二维和三 维的模拟将更容易观察 $\gamma^{\prime}$ 的形貌演化, 特别是其界面
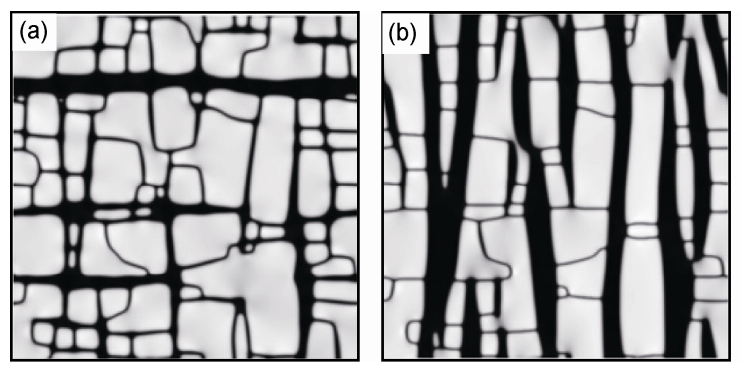

图 $4 \mathrm{Ni}_{82} \mathrm{Al}_{18}$ 合金不同条件下 $\gamma^{\prime}$ 形貌的对比 ${ }^{[14]}$

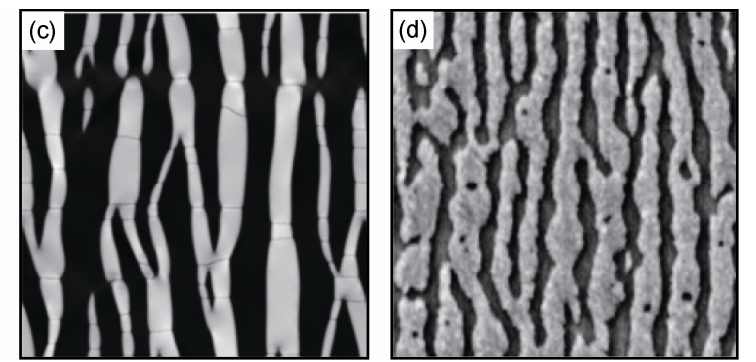

(a) $950^{\circ} \mathrm{C}$ 时效的相场模拟结果; (b) $950^{\circ} \mathrm{C}, 300 \mathrm{MPa}$ 蠕变的相场弹性模型的模拟结果; (c) $950^{\circ} \mathrm{C}, 300 \mathrm{MPa}$ 蠕变的相场弹性模型的 模拟结果; (d) 实验结果 
行为.

（2）将不同冷速及复杂热处理条件下多元镍基 单晶高温合金 $\gamma^{\prime}$ 形貌的演化考虑到模型中, 更好地探 究外场作用下 $\gamma^{\prime}$ 筏化行为.

（3）在二元黏塑性相场法研究 $\gamma^{\prime}$ 筏化的基础上, 借助黏塑性力学思路, 开展三元镍基高温合金黏塑 性条件下 $\gamma^{\prime}$ 筏化行为的研究.

\section{5 结束语}

热力学模型的进一步修正、CALPHAD 方法在相 场中的广泛应用以及弹塑性力学模型在相场法中的
完善, 将为相场法研究镍基单晶高温合金中 $\gamma^{\prime}$ 箋化行 为提供更广阔的前景. 通过耦合 CALPHAD 方法与 相场方法, 并将热动力学模型应用到相场方法中, 可 以解决多组元系统复杂热处理条件下的 $\gamma^{\prime}$ 析出问题, 使得相场法模拟近工况条件下镍基单晶高温合金 $\gamma^{\prime}$ 筏化行为成为可能, 形核理论在相场模型中的应用 将为模拟不同热处理条件下的 $\gamma^{\prime}$ 析出提供动力. 综合 应用弹塑性模型、计算相图方法、形核理论等将为实 现镍基单晶高温合金从前期热处理到后期蠕变、疲劳 条件下的 $\gamma^{\prime}$ 形貌演化及筏化行为的研究提供有力的 技术支撑.

\section{参考文献}

1 Nabarro F R N, Cress C M, Kotschy P. The thermodynamic driving force for rafting in superalloys. Acta Mater, 1996, 44: 3189-3198

2 Véron M, Brechet Y, Louchet F. Directional coarsening of Ni-based superalloys: Computer simulation at the mesoscopic level. Acta Mater, 1996, 44: 3633-3641

3 Boussinot G, Bour Y L, Finel A. Phase-field simulation with inhomogeneous elasticity: Comparison with atomic-scale method and application to superalloys. Acta Mater, 2010, 58: 4170-4178

4 Socrate S, Parks D M. Numerical determination of the elastic driving force for directional coarsening in Ni-superalloys. Acta Metall Mater, 1993, 41: 2185-2209

5 Zhou N, Shen C, Mills M J, et al. Modeling displacive-diffusional coupled dislocation shearing of $\gamma^{\prime}$ precipitates in Ni-base superalloys. Acta Mater, 2011, 59: 3484-3497

6 Vorontsov V A, Shen C, Wang Y, et al. Shearing of $\gamma^{\prime}$ precipitates by a $\left\langle\begin{array}{llll}1 & 1 & 2\end{array}\right\rangle$ dislocation ribbons in Ni-base superalloys: A phase field approach. Acta Mater, 2010, 58: 4110-4119

7 Kovarik L, Unocic R, Li J, et al. Microtwinning and other shearing mechanisms at intermediate temperatures in Ni-based superalloys. Prog Mater Sci, 2009, 54: 839-873

8 Paris O, Fahrmann M, Fahrmann E. Early stages of precipitate rafting in a single crystal Ni-Al-Mo model alloy investigated by small-angle X-ray scattering and TEM. Acta Mater, 1997, 45: 1085-1097

9 Gabb T P, Backman D G, Wei D Y, et al. $\gamma^{\prime}$ formation in a nickel-base disk superalloy. In: Pollock T M, Kissinger R D, Bowman R R, et al., eds. The 9th International Symposium on Superalloys, Champion, PA, 2000. Warrendale, PA: Minerals, Metals \& Materials Society, 2000. 405-414

10 Radis R, Schaffer M, Albu M, et al. Multimodal size distributions of $\gamma^{\prime}$ precipitates during continuous cooling of UDIMET 720 Li. Acta Mater, 2009, 57: 5739-5747

11 Sarosi P M, Wang B, Simmons J P, et al. Formation of multimodal size distributions of $\gamma^{\prime}$ in a nickel-base superalloy during interrupted continuous cooling. Scripta Mater, 2007, 57: 767-770

12 Leo P H, Lowenggrub J S, Jou H J. A diffuse interface model for microstructural evolution in elastically stressed solids. Acta Mater, 1998, 46: 2113-2130

13 Banerjee D, Banerjee R, Wang Y. Formation of split pattern of $\gamma^{\prime}$ precipitates in Ni-Al via particle aggregation. Scripta Mater, 1999, 41: 1023-1030

14 Gaubert A, Bouar Y L, Finel A. Coupling phase field and visco-plasticity to study rafting in Ni-base superalloys. Philos Mag A, 2010, 90: 375-404

15 Pareige-Schmuck C, Soisson F, Blavette D. Ordering and phase separation in low supersaturated Ni-Cr-Al alloys: 3D atom probe and Monte Carlo simulation. Mater Sci Eng A, 1998, 250: 99-103

16 赵彦, 陈铮, 王永欣, 等. 弹性应变能对 $\mathrm{Ni}_{75} \mathrm{Al}_{17} \mathrm{Zn}_{8}$ 合金过渡有序行为影响的微观相研究. 科学通报, 2010, 55: 182-187

17 Plapp M, Karma A. Eutectic colony formation: A stability analysis. Phys Rev E, 1999, 60: 6865-6889

18 Wang Y, Jin Y, Cuitino A, et al. A Phase field microelasticity theory and modeling of multiple dislocation dynamics. Appl Phys Lett, 2001, 78: 2324-2326

19 Athreya B, Goldenfeld N. Multiscale modeling of solidification: Phase-field methods to adaptive mesh refinement. Int J Modern Phys B, 2005, 19: 4525-4565 
20 Hu S Y, Chen L Q. Solute segregation and coherent nucleation and growth near a dislocation-A phase-field model integrating defect and phase microstructures. Acta Mater, 2001, 49: 463-472

21 Miehe C, Welschinger F, Hofacker H. A phase field model of electromechanical fracture. J Mech Phys Solids, 2010, 58: 1716-1740

22 Rubin G, Khachaturyan A G. Three-dimensional model of precipitation of ordered intermetallics. Acta Mater, 1999, 47: 1995-2002

23 Yamanaka A, Yoshihiro T, Tomita Y. Elastoplastic phase-field simulation of martensitic transformation with plastic deformation in polycrystal. Int J Mech Sci, 2010, 52: 245-250

24 Clayton J D, Knap J. A phase field model of deformation twinning: Nonlinear theory and numerical simulations. Phys D, 2011, 240: $841-858$

25 Zhou N, Shen C, Mills M J, et al. Phase field modeling of channel dislocation activity and $\gamma^{\prime}$ rafting in single crystal Ni-Al. Acta Mater, 2007, 55: 5369-5381

26 Zhou N, Shen C, Mills M, et al. Contributions from elastic inhomogeneity and from plasticity to $\gamma^{\prime}$ rafting in single-crystal Ni-Al. Acta Mater, 2008, 56: 6156-6173

27 Finel A, Le Bouar Y, Gaubert A, et al. Phase field methods: Microstructures, mechanics properties and complexity. C R Phys, 2010, 11: $245-256$

28 Artemev A, Jin Y, Khachaturyan A G. Three-dimensional phase field model of proper martensitic transformation. Acta Mater, 2001, 49: 1165-1177

29 Gong X F, Yang G X, Fu Y H, et al. First-principles study of Ni/Ni ${ }_{3} \mathrm{Al}$ interface strengthening by alloying elements. Comput Mater Sci, 2009, 47: 320-325

30 Kitashima T, Harada H. A new phase-field method for simulating $\gamma^{\prime}$ precipitation in multicomponent nickel-base superalloys. Acta Mater, 2009, 57: 2020-2028

31 Dupin N, Ansara I, Sundman S. Thermodynamic Re-assessment of the ternary system Al-Cr-Ni. Calphad-Comput Coupling Ph Diagrams Thermochem, 2001, 25: 279-298

32 Ansara I, Dupin N, Lukas H L, et al. Thermodynamic assessment of the Al-Ni system. J Alloy Compd, 1997, 247: 20-30

33 Kattner U R. The thermodynamic modeling of multicomponent phase equilibria. JOM, 1997, 49: 14-19

34 Wang Y, Banerjee D, Su C, et al. Field kinetic model and computer simulation of precipitation of $\mathrm{L}_{2}$ ordered intermetallics from fcc solid solution. Acta Mater, 1998, 46: 2983-3001

35 Vaithyanathan V, Chen L Q. 3D Simulation of coarsening of gamma-prime precipitates in a Ni-Al Alloy. In: Antonios G, Patrice E, Turchi A, et al., eds. Nucleation and Growth Processes in Materials, Boston, 1999. Masschusetts: MRS Press, 2000. $327-332$

36 Zhu J, Liu Z, Vaithyanathan V, et al. Linking phase-field model to CALPHAD: Application to precipitate shape evolution in Ni-base alloys. Scripta Mater, 2002, 46: 401-406

37 Wang J C, Osawa M, Yokokawa T, et al. Modeling the microstructural evolution of Ni-base superalloys by phase field method combined with CALPHAD and CVM. Comput Mater Sci, 2007, 39: 871-879

38 Kitashima T. Coupling of the phase-field and CALPHAD methods for predicting multicomponent, solid-state phase transformation. Philos Mag A, 2008, 88: 1615-1637

39 Kitashima T, Wang J C, Harada H. Phase-field simulation with the CALPHAD method for the microstructure evolution of multi-component Ni-base superalloys. Intermetallics, 2008, 16: 239-245

40 Gunton J D, Miguel M S, Sahni S. Phase Transitions and Critical Phenomena. New York: Academic Press, 1983

41 Simmons J P, Chen C, Wang Y. Phase field modeling of simultaneous nucleation and growth by explicitly incorporating nucleation events. Scripta Mater, 2000, 43: 935-942

42 Simmons J P, Wen Y, Shen C, et al. Microstructural development involving nucleation and growth phenomena simulated with phase field method. Mater Sci Eng A, 2004, 365: 136-143

43 Wen Y H, Simmons J P, Shen C, et al. Phase-field modeling of bimodal particle size distributions during continuous cooling. Acta Mater, 2003, 51: 1123-1132

44 Boussinot G, Finel A, Le Bouar Y. Phase-field modeling of bimodal microstructures in nickel-based superalloys. Acta Mater, 2009, 57: 921-931

45 Tien J K, Copley S M. The effect of uniaxial stress on the periodic morphology of coherent gamma prime precipitates in nickel-base super alloy single crystals. Metall Trans A, 1971, 2: 215-219

46 Véron M, Bréchet Y, Louchet F. Strain induced directional coarsening in Ni based superalloys. Scripta Mater, 1996, 34: 1883-1886

47 Pineau A. Influence of uniaxial stress on the morphology of coherent precipitates during coarsening-Elastic energy considerations. Acta Metall, 1976, 24: 559-564

48 Carry C, Strudel J L. Apparent and effective creep parameters in single crystals of a nickel base superalloy-II. Secondary creep. Acta Metall, 1978, 26: 859-870 
49 Miyazaki T, Nakamura K, Mori H. Experimental and theoretical investigations on morphological changes of $\gamma^{\prime}$ precipitates in Ni-Al single crystals during uniaxial stress-annealing. J Mater Sci, 1979, 14: 1827-1837

50 Fredholm A, Strudel J L. On the creep resistance of some nickel crystal. In: Gell M, Kortovich C S, Bricknell R H, et al., eds. The Proceeding of the 5th International Symposium on Superalloys, Champion, 1984. Champion: AIME Press, 1984. 211-220

51 Mughrabi H, Ott M, Tetzlaff U. New microstructural concepts to optimize the high-temperature strength of $\gamma^{\prime}$-hardened monocrystalline nickel-based superalloys. Mater Sci Eng A, 1997, 234-236: 434-437

52 Nathal M V, MacKay R A, Miner R V. Influence of precipitate morphology on intermediate temperature creep-properties of a nickel-base superalloy single-crystal. Metall Trans A, 1989, 20: 133-141

53 Schneider W, Hammer J, Mughrabi H. Creep deformation and rupture behaviour of the monocrystalline superalloy CMSX-4-A comparison with the alloy SRR-99. In: Antolovich S D, Stusrud R W, Mackay R A, et al., eds. The Proceeding of the 7th International Symposium on Superalloys, Champion, PA, 1992. Warrendale, PA: Minerals, Metals \& Materials Society, 1992. 589-598

54 Pearson D D, Lemkey F D, Kear B H. Stress coarsening of $\gamma^{\prime}$ and its influence of creep properties of a single crystal superalloy. In: Wloder S T, Danesi W P, Kent W B, et al., eds. The Proceeding of the 4th International Symposium on Superalloys. Champion: ASM Press, 1980. 513-520

55 Pollock T M, Argon A S. Directional coarsening in nickel-base single crystals with high volume fractions of coherent precipitates. Acta Mater, 1994, 42: 1859-1874

56 Pollock T M, Argon A S. Creep resistance of CMSX-3 nickel base superalloy single crystals. Acta Metall Mater, 1992, 40: 1-30

57 Ignat M, Bufere J, Chaix J. Microstructures induced by a stress gradient in a nickel-based superalloy. Acta Metall Mater, 1993, 41: $855-862$

58 Matan N, Cox D C, Rae C M F, et al. On the kinetics of rafting in CMSX-4 superalloy single crystals. Acta Mater, 1999, 47: 2031-2045

59 Fahrmann M, Hermann W, Fahrmann E, et al. Determination of matrix and precipitate elastic constants in $\left(\gamma-\gamma^{\prime}\right)$ Ni-base model alloys, and their relevance to rafting. Mater Sci Eng A, 1999, 260: 212-221

60 Osawa M, Shiraishi H, Yokokawa T, et al. 3D-FEM calculations of rafting in Ni-base superalloys based on high temperature elastic and lattice parameters. In: Green K A, Pollock TM, Harada H, er al., eds. The 10th International Symposium on Superalloys. Warrendale, PA: Minerals, Metals \& Materials Society, 2004. 977-985

61 Lee J K. Coherency strain analyses via a discrete atom method. Scripta Mater, 1995, 32: 559-564

62 Nabarro F R N. The chemical driving force for rafting in superalloys. Scripta Mater, 1997, 37: 497-501

63 Buffiere J Y, Ignat M. A dislocation based criterion for the raft formation in nickel-based superalloys single crystals. Acta Metall Mater, 1995, 43: 1791-1797

64 Arrell D, Vallés J. Rafting prediction criterion for superalloys under a multiaxial stress. Scripta Mater, 1996, 35: 727-732

65 Vallés J, Arrell D. Monte Carlo simulation of anisotropic coarsening in nickel-base superalloys. Acta Mater, 42: 2999-3008

66 Ohashi T, Hidaka K, Saito M. Quantitative study of the plastic slip deformation and formation of internal stresses in Ni-base superalloys. Mater Sci Eng A, 1997, 238: 42-49

67 Ohashi T, Hidaka K, Saito M. Elastic stress in single crystal Ni-base superalloys and the driving force for their microstructural evolution under high temperature creep conditions. Acta Mater, 1997, 45: 1801-1810

68 Khachaturyan A G. Theory of Structural Transformation in Solids. New York: Wiley Press, 1983

69 Mccormack M, Khachaturyan A G, Morris J W. A two-dimensional analysis of the evolution of coherent precipitates in elastic media. Acta Metall Mater, 1992, 40: 325-336

70 Khachaturyan A G, Semenovakays S, Tsakalakos T. Elastic strain energy of inhomogeneous solids. Phys Rev B, 1995, 52: 15909-15919

71 Hu S Y, Chen L Q. A phase-field model for evolving microstructures with strong elastic inhomogeneity. Acta Mater, 2001, 49: 1879-1890

72 Zhu J Z, Chen L Q, Shen J. Morphological evolution during phase separation and coarsening with strong inhomogeneous elasticity. Model Simul Mater Sci Eng A, 2001, 9: 499-511

73 Chen L Q, Shen J. Applications of semi-implicit Fourier-spectral method to phase field equations. Comput Phys Commun, 1998, 108: $147-158$

74 Zhu J Z, Chen L Q. Coarsening kinetics from a variable-mobility Cahn-Hilliard equation: Application of a semi-implicit Fourier spectral method. Phys Rev E, 1999, 60: 3564-3572

75 Wang Y Z, Khachaturyan A G. Microstructural evolution during the precipitation of ordered intermetalllics in multiparticle coherent system. Philos Mag A, 1995, 72: 1161-1171

76 Li D Y, Chen L Q. Shape evolution and splitting of coherent particles under applied stresses. Acta Mater, 1999, 47: 247-257

77 Yang P Y, Li S C, Zheng Z Q, et al. Precipitation and coarsening of coherent and ordered second phase during stress aging by using phase-field simulations. Rare Metal Mater Eng, 2007, 36: 1341-1345 
78 Véron M, Bastie P. Strain induced directional coarsening in nickel based superalloys: Investigation on kinetics using the small angle neutron scattering (SANS) technique. Acta Mater, 1997, 45: 3277-3282

79 Rodney D, Bounar Y Le, Finel A. Phase field methods and dislocations. Acta Mater, 2003, 51: 17-30

80 Yeh A C, Rae C, Tin S. High temperature creep of Ru-bearing Ni-base single crystal superalloy. In: Green K A, Pollock T M, Harada H, et al., eds. The 10th International Symposium on Superalloys. Warrendale, PA: Minerals, Metals \& Materials Society, 2004. 677-685

81 Tetzlaff U, Mughrabi H. Enhencement of the high-temperature tensile creep strength of monocrystalline nickel-base superalloys by prerafting in compression. In: Pollock T M, Kissinger R D, Bowman R R, et al., eds. The 9th International Symposium on Superalloys. Warrendale, PA: Minerals, Metals \& Materials Society, 2000. 273-282

82 Lahrman D, Field R, Darolia R, et al. Investigation of techniques for measuring lattice mismatch in a rhenium containing nickel base superalloy. Acta Metall, 1988, 36: 1309-1320

83 Singh A K, Louat N, Sadanana K. Dislocation network formation and coherency loss around gamma-prime precipitates in a nickel-base superalloy. Metall Mater Trans A, 1988, 19: 2965-2973

84 Reed R, Matan N, Cox D, et al. Creep of CMSX-4 superalloy single crystals: Effects of rafting at high temperature. Acta Mater, 1999, 47: 3367-3381

85 Keller R, Maier H, Mughrabi H. Characterization of interfacial dislocation networks in a creep-deformed nickel-base superalloy. Scripta Metall Mater, 1993, 28: 23-28

86 Probst-Hein M, Dlouhy A, Eggeler G. Dislocation interactions in $\gamma$-channels between $\gamma^{\prime}$-particles of superalloy single crystals. Mater Sci Eng A, 2001, 319-321: 379-382

87 Dlouhy A, Probst-Hein M, Eggeler G. Static dislocation interactions in thin channels between cuboidal particles. Mater Sci Eng A, 2001, 309-310: 278-282

88 Shen C. The fundamentals and applications of phase field method in quantitative microstructural modeling. Dissertation for Doctor Degree. Columbus: The Ohio State University, 2004

89 Léonard F, Desai R C. Spinodal decomposition and dislocation lines in thin films and bulk materials. Phys Rev B, 1998, 58: 8277-8288

90 Carry C, Dermarkar S, Strudel J, et al. Internal stresses due to dislocation walls around second phase particles. Metall Mater Trans A, 1979, 10: 855-860

91 Ubachs R L J M, Schreurs P J G, Geers M G D. Phase field dependent viscoplastic behaviour of solder alloys. Int J Solids Struct, 2005, 42: 2533-2558

92 Uehara T, Tsujino T, Ohno N. Elasto-plastic simulation of stress evolution during grain growth using a phase field model. J Cryst Growth, 2007, 300: 530-537

93 Guo X, Shi S, Zhang Q, et al. An elastoplastic phase-field model for the evolution of hydride precipitation in zirconium. Part II: Specimen with flaws. J Nucl Mater, 2008, 378: 120-125 